\title{
Clarification of the link between polyunsaturated fatty acids and Helicobacter pylori-associated duodenal ulcer disease: a dietary intervention study
}

\author{
BY A. E. DUGGAN ${ }^{1}$, J. C. ATHERTON ${ }^{1}$, A. COCKAYNE ${ }^{2}$, M. BALSITIS $^{3}$, S. EVISON $^{4}$, \\ T. HALE ${ }^{1}$, C. J. HAWKEY ${ }^{1}$ AND R. C. SPILLER ${ }^{1}$ \\ ${ }^{1}$ Division of Gastroenterology, ${ }^{2}$ Institute of Infection and Immunity, ${ }^{3}$ Department of Histopathology, and \\ ${ }^{4}$ Department of Dietetics, University Hospital, Nottingham NG7 $2 U H$
}

(Received 17 February 1997 - Accepted 19 March 1997)

\begin{abstract}
Epidemiological evidence has suggested that the declining prevalence of duodenal ulcer disease may be attributable to rising consumption of polyunsaturated fatty acids, a hypothesis supported by in vitro evidence of toxicity of such substances to Helicobacter pylori. The objective of the present study was to establish whether this association is causal. Forty patients with proven infection with $H$. pylori and endoscopic evidence of past or present duodenal ulcer disease were randomized to receive either polyunsaturated fatty acids (PUFA group), in the form of capsules and margarine, or a placebo (control). Both groups received concurrent $\mathrm{H}_{2}$ antagonist therapy. Efficacy of therapy was determined endoscopically by assessment of ulcer healing while $\boldsymbol{H}$. pylori status was determined by antral biopsy, urease (EC 3.5.1.5) culture and histological assessment of the severity of $H$. pylori infection. Antral levels of prostaglandin $\mathrm{E}_{2}\left(\mathrm{PGE}_{2}\right)$ and leukotriene $\mathrm{B}_{4}\left(\mathrm{LTB}_{4}\right)$ were quantified. Compliance was monitored. Before treatment, both groups were comparable for severity of $H$. pylori infection, smoking status and levels of $\mathrm{LTB}_{4}$ and $\mathbf{P G E}_{2}$. Despite a significant difference in consumption of linoleic acid (19.9 (SE 1.6) $\mathrm{g}$ for PUFA group $v .6 .7$ (SE 0.8$) \mathrm{g}$ for controls $(P<0.01)$ and linolenic acid (2.6 (SE 0.2) $\mathrm{g} v .0 .6$ (SE 0.03) $\mathrm{g}(P<0.01)$ there was no significant change in either the severity of $H$. pylori infection or prostaglandin levels in either group at 6 weeks. Consumption of a considerable amount of PUFA does not inhibit the colonization of the stomach by $H$. pylori nor does this alter the inflammatory changes characteristic of $\boldsymbol{H}$. pylori gastritis. We conclude that the association between duodenal ulceration and a low level of dietary PUFA is likely to be spurious, probably reflecting the effect of confounding factors such as affluence, social class or smoking.
\end{abstract}

Helicobacter pylori: Polyunsaturated fatty acids: Duodenal ulcer

It is about 10 years since it was noted that the continuing decline in peptic ulcer morbidity and mortality in, at least, the UK and the USA was associated with a rising consumption of polyunsaturated fatty acids (PUFA) (Hollander \& Tarnawski, 1986). A plausible hypothesis to relate these two phenomena was supported by a number of in vitro and in vivo studies which suggested that dietary PUFA inhibited gastric acid secretion while increasing cytoprotective prostaglandins (Hollander et al. 1982; Tarnawski et al. 1987; Grant et al. 1988, 1990; Hunt et al. 1988). It is now well recognized that prostaglandins, plentiful throughout the gastrointestinal mucosa, play a major role in gastric cytoprotection, increasing mucosal blood flow, decreasing gastric acid secretion, stimulating mucus secretion, and preventing gastric mucosal barrier disruption through altering transmucosal potential difference and mucosal permeability (Robert et al. 1979; Miller, 1983). Inhibitors of prostaglandin synthesis are also known to exert a deleterious effect on the gastric mucosa. Dietary essential fatty acids, particularly linoleic acid, a precursor for arachidonic 
acid and prostaglandins, have been demonstrated to increase gastric prostaglandin levels after acute dosing and to modify the gastric mucosal injury induced by a number of toxic substances (Miller, 1983). Helicobacter pylori is one such agent toxic to the gastric mucosa. It has been shown to lead to thinning of the mucus layer and to diminish the hydrophobicity of the mucosal surface (Spychal et al. 1990; Goggan et al. 1992; Mauch et al. 1993). Recently linoleic acid has been shown to inhibit $H$. pylori growth in vitro (Thompson et al. 1994). Together these studies suggest that essential fatty acids, particularly linoleic acid, by a variety of mechanisms, may be important in the inhibition of $H$. pylori-induced peptic ulceration. The clinical significance of these findings is suggested by epidemiological studies which have revealed a lower concentration of PUFA in the adipose tissue of duodenal ulcer patients; this is an indirect measure of the long-term average fatty acid composition of the diet (Hollander \& Tarnawski, 1986; Staveren et al. 1986; Kearney et al. 1989). Despite this, and the otherwise unexplained downward trend in duodenal ulcer disease, no in vivo trials of PUFA therapy in $H$. pylori-positive duodenal ulcer patients have been published. Given the extremely high prevalence of $H$. pylori infection and the impracticability of pharmacological treatment for entire populations, the discovery of a dietary method of modifying consequences of $H$. pylori infection would be extremely valuable. This led us to undertake a direct study of the effect of increasing the intake of PUFA.

\section{METHODS}

Forty volunteer patients referred to a routine endoscopy clinic with infection with $H$. pylori and either past or present duodenal ulcer disease, who had not received antibiotics, bismuth or omeprazole within the previous 2 months, were eligible for enrolment in the study. At endoscopy, evidence of duodenal ulcer disease (erosive duodenitis, duodenal scarring, active duodenal ulcer disease) was documented by the endoscopist. An active ulcer was defined as a mucosal breach $>5 \mathrm{~mm}$ in one dimension with depth and an erosion as a break in the mucosal surface not fulfilling the stated criteria. Scarring was defined as distortion and pitting of the duodenum with or without an ulcer scar. Histological assessment was performed using a modified Sydney system of classification of density of $H$. pylori infection where both mild and severe categories of infection were each subdivided into two further categories. The categories were: (1) occasional organisms identified; (2) groups of organisms, up to one-third of the mucosal surface; (3) organisms covering between one-third and two-thirds of the surface; (4) organisms covering more than two-thirds of the surface and upper pits; (5) organisms covering more than two-thirds of the surface and upper pits, and dense groups of organisms. Density of infection was defined as the cumulative total of the grades of one antral and one body biopsy. $H$. pylori infection was demonstrated by a urease (EC 3.5.1.5) test (CLO test, Delta West Pty Ltd, Bentley, Australia), histology of two biopsies from the body and antrum, and culture of antral biopsies. Patients were considered $H$. pylori infected if two or more tests were positive. All patients were $H$. pylori positive according to these criteria. Prostaglandin $\mathrm{E}_{2}$ $\left(\mathrm{PGE}_{2}\right)$ and leukotriene $\mathrm{B}_{4}\left(\mathrm{LTB}_{4}\right)$ content of the biopsies was estimated by radioimmunoassay (Amersham TRK 431, Amersham, Bucks.) which we have previously validated (Hawkey, 1986).

Patients were then randomized in a double-blind manner to a $35 \mathrm{~d}$ treatment of either placebo capsules (coconut oil and vitamin E, Scotia Pharmaceuticals, Guildford, Surrey) and a low-PUFA margarine (Summer County, J.S. Sainsbury plc, Stamford St, London) or a high-PUFA regimen in the form of Efamol ${ }^{\circledR}$ capsules (Scotia Pharmaceuticals; four 
Table 1. Characteristics of subjects with Helicobacter pylori infection who were assigned to receive a high-polyunsaturated fatty acid diet (PUFA group) or a control diet

\begin{tabular}{lcc}
\hline \hline & PUFA group & Control group \\
\hline Sex (M:F) & $14: 6$ & $16: 4$ \\
Age (years) & 49 (SE 13) & $56($ SE 11) \\
Smoking status & $9(45)$ & $9(45)$ \\
No. of smokers (\%) & $6(30)$ & $6(30)$ \\
No. of non-smokers (\%) & $2(10)$ & $4(20)$ \\
No. of ex-smokers (\%) & $3(15)$ & $1(5)$ \\
No. unknown (\%) & $12: 6: 3^{*}$ & $7: 10: 6^{*}$ \\
\hline Duodenal ulcer:Scar:Duodenitis & \\
\hline \hline
\end{tabular}

* Dual pathology (one patient, scar + duodenal ulcer; three patients, scar + duodenitis).

Table 2. Sources of essential fatty acids supplied to subjects with Helicobacter pylori infection assigned to receive a high-polyunsaturated fatty acid diet (PUFA group) or a control diet

\begin{tabular}{|c|c|c|c|c|}
\hline & \multicolumn{2}{|c|}{ Linoleic acid } & \multicolumn{2}{|c|}{ Linolenic acid } \\
\hline & PUFA group & Controls & PUFA group & Controls \\
\hline \multicolumn{5}{|l|}{ Capsules } \\
\hline (g/dose) & 1.3 & nil & 0.2 & nil \\
\hline$(\mathrm{g} / \mathrm{d})$ & $5 \cdot 2$ & nil & 0.8 & nil \\
\hline Margarine ( $\mathrm{g} / \mathrm{kg}$ margarine) & 397 & 68 & 56 & 3 \\
\hline
\end{tabular}

$500 \mathrm{mg}$ capsules four times daily) and a high-PUFA margarine (Soya, J.S. Sainsbury plc). Capsules were taken with or after meals and the margarine was used in place of the subjects' normal spread or oil in cooking. Characteristics of the two groups are outlined in Table 1. Both groups received a concurrent $\mathrm{H}_{2}$ antagonist, ranitidine (150 $\mathrm{mg}$ twice daily), as initial treatment for their dyspepsia. The PUFA content of the capsules and spreads provided to the two treatment groups is outlined in Table 2.

A $\left[{ }^{14} \mathrm{C}\right]$ urea breath test was performed before commencing therapy. Patients fasted overnight and were then given a fat-containing meal (Calogen, Scientific Hospital Supplies Ltd, Liverpool, Merseyside) to delay gastric emptying, just before drinking $50 \mathrm{ml}$ water containing the ${ }^{14} \mathrm{C}$-labelled urea. The $\left[{ }^{14} \mathrm{C}\right]$ urea breath test involves administration of $46 \mathrm{kBq}\left[{ }^{\mathrm{f}} \mathrm{C}\right.$ ] ${ }^{2}$ rea which is split by $\mathrm{H}$. pylori in the stomach to ammonium and bicarbonate ions, which, in the presence of acid, yield ${ }^{14} \mathrm{CO}_{2}$. Breath is collected and the amount of ${ }^{14} \mathrm{CO}_{2}$ expired is measured. The $\left[{ }^{14} \mathrm{C}\right]$ urea is delivered orally in $50 \mathrm{ml}$ water. The results are reported as the percentage of the dose excreted $/ \mathrm{mmol} \mathrm{CO}_{2}$ at $30 \mathrm{~min}$ post-dosing. All other ulcer healing medications, antibiotics, aspirin, and non-steroidal anti-inflammatory drugs were proscribed. Compliance was assessed by tablet count, review of concurrent medications on follow-up visits, diary card, weighing of the returned margarine containers and review by a dietitian blinded to the randomization at the time of assessment. Each patient on entry to the study was given a daily diary form and asked to tick or cross the four treatment times each day according to whether they had or had not taken their PUFA or ranitidine capsules. Patients were asked to return these forms and all capsule bottles at their follow-up visit on day 30 . On this occasion they were given a food diary and asked to record their total diet including quantities of each food consumed for a $3 \mathrm{~d}$ period then bring the diary with them to the dietitian for calculation of daily PUFA consumption. 
At the end of the treatment period patients underwent repeat endoscopy and biopsy assessments as undertaken at the commencement of the trial. A repeat $\left[{ }^{14} \mathrm{C}\right]$ urea breath test was performed and consumption of PUFA was assessed. Ethical approval was obtained from the hospital ethics committee.

\section{Statistical methods}

PUFA and control group results were all nonparametric and were compared using the Wilcoxon Signal Rank test for paired data and the Mann-Whitney U test for unpaired data.

\section{RESULTS}

Twenty patients received the active treatment and twenty the placebo. One patient from the placebo group was lost to follow-up. All treatments were well tolerated, with no significant adverse events in either group. Compliance, defined as $80 \%$ or greater consumption of ranitidine tablets was $95 \%$ in cases and $90 \%$ in controls, with consumption unknown in two cases and four controls. Table 3 shows the mean consumption of PUFA for the two groups according to dietary assessment of $3 \mathrm{~d}$ food charts and calculation of consumption of PUFA from returned capsules and margarine. As can be seen, mean total daily intake of PUFA from all sources (dietary + margarine + capsules) in the PUFA group was 19.9 (SE 1.6) $\mathrm{g}$ linoleic acid and 2.6 (SE 0.2) $\mathrm{g}$ linolenic acid compared with 6.7 (SE 0.8) $\mathrm{g}$ linoleic acid and 0.6 (SE 0.03) $\mathrm{g}$ linolenic acid in the controls.

For six subjects (one case and five controls) compliance was unknown, as capsules were not returned. For a further case, dietary assessment was not available. $\left[{ }^{14} \mathrm{C}\right]$ urea breath test results are shown in Fig. 1. There was no statistically significant difference in the breath test results at $30 \mathrm{~min}$ between cases and controls before treatment $(P=0 \cdot 14)$. Treatment was not shown to be of benefit to either cases or controls $(P=0.48)$. In cases there was a trend to higher $\left[{ }^{14} \mathrm{C}\right]$ urea breath test results after treatment $(P=0.006)$.

$\mathrm{PGE}_{2}$ levels showed no statistically significant change with treatment in either cases or controls (cases: before treatment, median 75.3, interquartile range 63.4-120.0; after treatment, median 89.8, interquartile range 62.2-136.6 $(P=0.79)$; controls: before treatment, median 73.3, interquartile range 59.2-106.0; after treatment, median 64.5, interquartile range 33.4-103.9 $(P=0.62)$. $\mathrm{LTB}_{4}$ levels were also not significantly affected

Table 3. Daily consumption of polyunsaturated fatty acids (PUFA) by subjects with Helicobacter pylori infection assigned to receive a high-PUFA diet (PUFA group) or a control diet*

(Mean values with their standard errors for twenty subjects per group)

\begin{tabular}{|c|c|c|c|c|c|c|c|c|}
\hline \multirow{3}{*}{$\begin{array}{l}\text { Daily PUFA } \\
\text { consumption (g) }\end{array}$} & \multicolumn{4}{|c|}{ PUFA group } & \multicolumn{4}{|c|}{ Control group } \\
\hline & \multicolumn{2}{|c|}{ Linoleic acid } & \multicolumn{2}{|c|}{ Linolenic acid } & \multicolumn{2}{|c|}{ Linoleic acid } & \multicolumn{2}{|c|}{ Linolenic acid } \\
\hline & Mean & SE & Mean & SE & Mean & SE & Mean & SE \\
\hline Dietary estimation & 4.7 & 0.7 & 0.6 & 0.1 & $4 \cdot 3$ & 0.8 & 0.5 & 0.1 \\
\hline Calculation from returned capsules & 4.7 & 0.1 & 0.6 & 0.02 & nil & & nil & \\
\hline Calculation from returned margarine & $10 \cdot 6$ & $1 \cdot 3$ & 1.4 & 0.2 & $2 \cdot 3$ & $0 \cdot 3$ & 0.1 & 0.01 \\
\hline Total & 19.9 & 1.6 & $2 \cdot 6$ & 0.2 & 6.7 & 0.8 & 0.6 & 0.03 \\
\hline
\end{tabular}

* For details of diets and procedures, see pp. 516-518. 

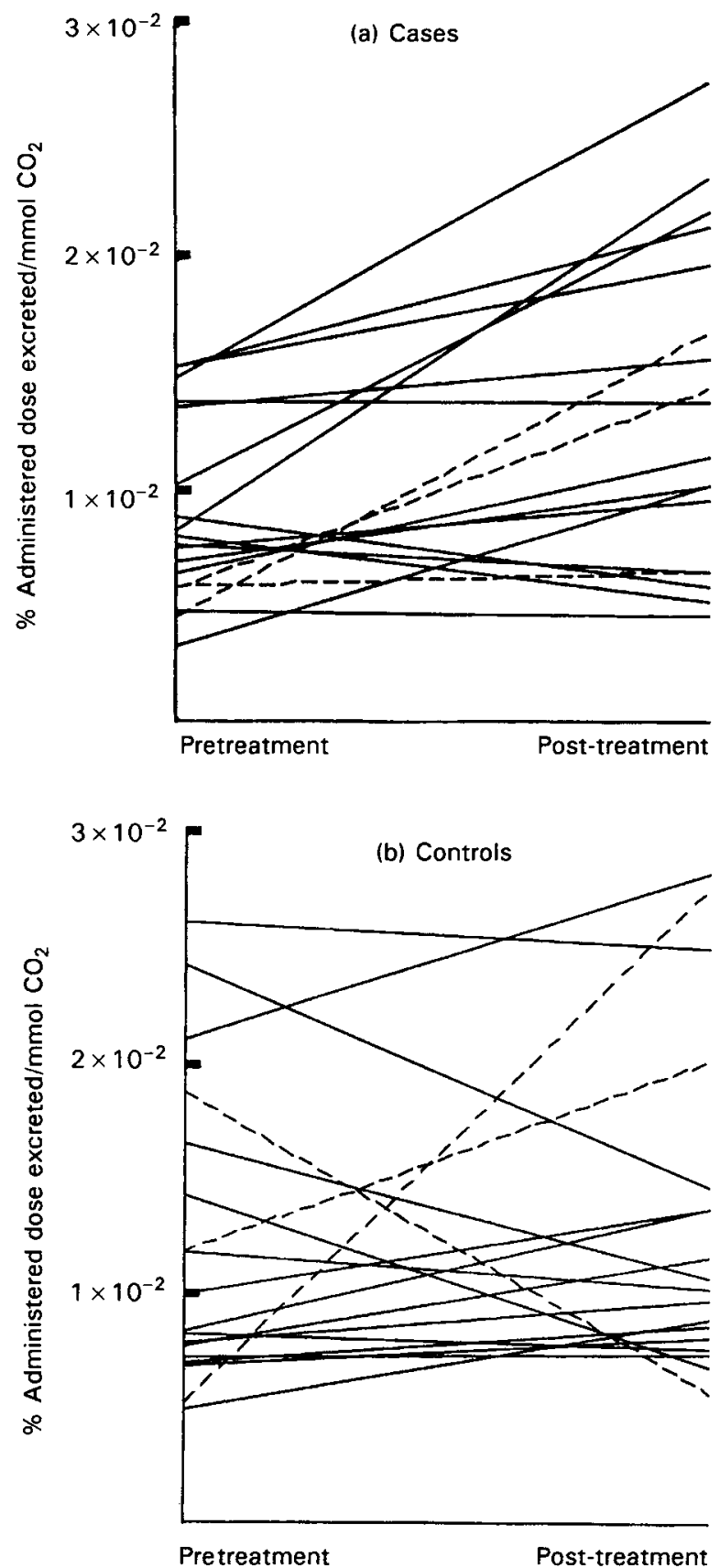

Fig. 1. Excretion of ${ }^{14} \mathrm{CO}_{2}$ during a $\left[{ }^{14} \mathrm{C}\right]$ urea breath test by subjects with Helicobacter pylori infection, before and after $35 \mathrm{~d}$ treatment with a high-polyunsaturated fatty acid diet (cases) or a control diet (controls). For details of procedures, see p. 517. 
by treatment in either cases or controls (cases: before treatment, median 0.50 , interquartile range $0.38-1.3$; after treatment, median 0.55 , interquartile range $0.39-0.84(P=0.77)$; controls: before treatment, median 0.53 , interquartile range $0.40-1.86$; after treatment, median 0.53 , interquartile range $0.40-0.77(P=0.95))$. Treatment reduced $H$. pylori density in the cases, but this was not significant (before treatment, median 4 , interquartile range 3-4; after treatment, median 3 , interquartile range $2-5 ;(P=0.07)$. There was also a decrease in $H$. pylori density in the placebo group which did not reach significance (before treatment, median 6 , interquartile range $4-7$; after treatment, median 4 , interquartile range 3-6 $(P=0.06)$.

\section{DISCUSSION}

Several authors have noted a declining prevalence of peptic ulcer in the UK and the USA in the last almost 30 years (Coggan et al. 1981; Fineberg \& Pearlman, 1981; Sonnenberg $e t$ al. 1985). A fall in the rate of surgery for peptic ulcer disease, at least in the USA, occurred before the introduction of cimetidine, the first of the $\mathrm{H}_{2}$ antagonists. This suggests that other factors have altered the prevalence, if not the severity, of the disease in this period (Fineberg \& Pearlman, 1981). The association of poor living standards with increased $H$. pylori prevalence and in turn $H$. pylori infection with peptic ulcer disease is becoming increasingly clear. Improving hygiene is also associated with improvements in nutrition and, historically at least, there is a clear relationship between increased dietary PUFA intake and declining duodenal ulceration (Hollander \& Tarnawski, 1986).

In previous work we and others have shown that incubation of $H$. pylori microaerophylically with a range of PUFA has an inhibitory effect on $H$. pylori (Thompson et al. 1994). In studies with $\omega$-linolenic acid at concentrations of $10^{-3} \mathrm{M}$ virtually all organisms were killed. Electron microscopy of $H$. pylori shows disruption of the cell membrane to be the mechanism of cell lysis (Maczulak et al. 1981; Thompson et al. 1994). At lower concentrations of these PUFA, inhibition of $H$. pylori motility has been observed (Thompson et al. 1994; Khulusi, 1995). While the mechanism remains unclear, animal studies have shown that long-chain fatty acids inhibit rumen bacterial metabolism (Maczulak et al. 1981). Our own studies have shown a relationship between the degree of unsaturation and the antibacterial effect against human colonic bacteria (Thompson et al. 1992).

PUFA also may be effective against $H$. pylori by stimulating prostaglandin production. Prostaglandins of the E, A, and I series have been shown to suppress basal and stimulated gastric acid secretion in rats and normal human subjects (Hollander et al. 1982). Furthermore, it has been demonstrated that prostaglandins of the E type are capable of preventing formation of gastric ulcers in rats induced by a wide variety of experimental techniques (Robert et al. 1979). Tarnawski et al. (1987) also showed a significant cytoprotective effect of oral administration of linoleic acid on ethanol-induced gastric injury, an effect significantly reduced by pretreatment with oral indomethacin, a known inhibitor of prostaglandin sythetase.

Our present study failed to show an effect of PUFA consumption on $H$. pylori gastritis which underlies most duodenal ulcer disease, despite levels of PUFA in excess of that which would be accounted for in a normal diet. While our own work in vitro, and that of other authors, has shown both toxicity of PUFA to $H$. pylori and a trophic effect on gastric prostanoid levels, our current findings suggest that these are not clinically significant effects even with a high-PUFA diet. One reason may be that PUFA are rarely ingested in 
isolation. Their effect may, therefore, be attenuated by binding to other foods consumed simultaneously.

Unlike Grant et al. (1988) we failed to find any effect of substantial PUFA intake on gastric prostaglandin production. This difference is likely to be due to different methodologies. While Grant et al. (1988) assayed PGE levels from aspirated gastric juice collected over $75 \mathrm{~min}$, in the present study levels were derived from biopsies. The reliability of this method is well established and is therefore unlikely to explain our failure to show a significant rise in PGE levels (Hawkey, 1986). More importantly, Grant et al. (1988) studied gastric $\mathrm{PGE}_{2}$ production $1 \mathrm{~h}$ after linoleic acid intake and noted the effect to be declining over the $1 \mathrm{~h}$. This implies a very acute, short-lived response which we would have missed since our subjects had taken no PUFA for at least $8 \mathrm{~h}$ overnight.

The association of PUFA with the decline in peptic ulcer incidence may be an epiphenomenon. In studies of adipose fatty acid profiles, which reflect dietary fat intake over the preceding 9-12 months, duodenal ulcer patients have been found to have a lower mean percentage of linoleic acid than controls (Grant et al. 1990). Using the virtually $1: 1$ relationship between percentage linoleic acid in adipose tissue and percentage in the diet (Staveren et al. 1986) we can predict that the differences in PUFA intake between ulcer patients and controls in the study of Grant et al. (1990) would be of the order of $30 \%$. However in our studies we increased the PUFA intake $400 \%$ and yet noted no difference in either mucosal prostaglandin levels or $H$. pylori colonization. More detailed analysis of dietary intake has suggested that low PUFA intake may simply be a marker for other dietary and social habits including high salt intake and increased rates of smoking (Oliver, 1989). Furthermore, smoking may lower tissue levels of linoleic acid by altering fatty acid metabolism. Controlling for the effects of smoking greatly reduces the difference in incidence of low PUFA levels in duodenal ulcer patients (Grant et al. 1990). Such a difference may in fact be indicative of a broader variation in the diet of duodenal ulcer patients as compared with controls. This hypothesis warrants further investigation but the present study suggests that merely changing intake of PUFA will not alter the risk of developing a $H$. pylori-associated duodenal ulcer.

\section{REFERENCES}

Carriere, F., Barrowman, J., Verger, R. \& Laugier, R. (1993). Secretion and contribution to lipolysis of gastric and pancreatic lipases during a test meal in humans. Gastroenterology 105, 876-888.

Coggan, D., Lambert, P. \& Langman, M. J. S. (1981). 20 Years of hospital admissions for peptic ulcer in England and Wales. Lancet i, 1302-1305.

Fineberg, H. \& Pearlman, L. A. (1981). Surgical treatment of peptic ulcer in the United States. Trends before and after the introduction of cimetidine. Lancet i, 1305-1307.

Goggan, P., Marrero, J., Spychal, R., Jackson, P., Corbishley, C. \& Northfield, T. (1992). Surface hydrophobicity of gastric mucosa in Helicobacter pylori infection: effect of clearance and eradication. Gastroenterology 103, 1486-1490.

Grant, H., Palmer, K., Kelly, R., Wilson, N. \& Misiewicz, J. (1988). Dietary linoleic acid and prostaglandin secretion. Gastroenterology 94, 955-959.

Grant, H. W., Palmer, K. R., Riermesma, R. R. \& Oliver, M. F. (1990). Duodenal ulcer is associated with low dietary linoleic acid intake. Gut 31, 997-998.

Hawkey, C. J. (1986). Synthesis of prostaglandins $E_{2}$ and thromboxane $B_{2}$ and prostaglandin catabolism in gastritis and gastric ulcer. Gut 27, 1484-1492.

Hollander, D. \& Tarnawski, A. (1986). Dietary essential fatty acids and decline in peptic ulcer disease - a hypothesis. Gut 27, 239-242.

Hollander, D., Tarnawski, A., Ivey, K., DeZeery, A., Zipser, R., McKenzie, W. \& McFarland, W. (1982). Arachidonic acid protection of rat gastric mucosa against ethanol injury. Journal of Laboratory and Clinical Medicine 100, 296-308.

Hunt, M., Hillier, K. \& Jewell, R. (1988). Modification of upper gastrointestinal prostaglandin synthesis by dietary fatty acids. Prostaglandins 35, 597-608. 
Kearney, J., Kennedy, N. P., Keeling, P. W. N., Keating, J. J., Grubb, L., Kennedy, M. \& Gibney, M. J. (1989). Dietary intakes and adipose tissue levels of linoleic acid in peptic ulcer disease. British Journal of Nutrition 62, 699-706.

Khulusi, S. (1995). Duodenal ulceration. Pathogenesis and the role of Helicobacter pylori. MD Thesis submitted to the Faculty of Medicine, University of Bristol.

Maczulak, A. E., Dehority, B. A. \& Palmquist, D. L. (1981). Effects of long-chain fatty acids on growth of rumen bacteria. Applied Microbiology 42, 856-862.

Mauch, F., Bode, G., Ditschuneit, H. \& Malfertheiner, P. (1993). Demonstration of a phospholipid-rich zone in the human gastric epithelium damaged by Helicobacter pylori. Gastroenterology 105, 1698-1704.

Miller, T. (1983). Protective effects of prostaglandins against gastric mucosal damage: current knowledge and proposed mechanisms. American Journal of Physiology 245, G601-G623.

Oliver, M. F. (1989). Cigarette smoking, polyunsaturated fats, linoleic acid, and coronary heart disease. Lancet $\mathbf{i}$, 1241-1242.

Robert, A., Nezamis, J., Lancaster, C. \& Hanchar, A. (1979). Cytoprotection by prostaglandins in rats. Gastroenterology 77, 433-443.

Sonnenberg, A., Muller, H. \& Fabio, P. (1985). Birth-cohort analysis of peptic ulcer mortality in Europe. Journal of Chronic Diseases 38, 309-317.

Spychal, R., Groggan, P., Marrero, J. M., Saverymuttu, S., Yu, C., Corbishley, C., Maxwell, J. \& Northfield, T. (1990). Surface hydrophobicity of gastric mucosa in peptic ulcer disease. Relationship to gastritis and Campylobacter pylori infection. Gastroenterology 98, 1250-1254.

Staveren, W., Deurenberg, P., Katan, M., Burema, J., de Groot, L. \& Hoffmans, A. (1986). Validity of the fatty acid composition of subcutaneous fat tissue microbiopsies as an estimate of the long term average fatty acid composition of the diet of separate individuals. American Journal of Epidemiology 123, 455-463.

Tarnawski, A., Hollander, D. \& Gergely, H. (1987). Protection of the gastric mucosa by linoleic acid - a nutrient essential fatty acid. Clinical and Investigative Medicine 3, 132-135.

Thompson, L., Cockayne, A. \& Spiller, R. C. (1994). Inhibitory effect of polyunsaturated fatty acids on the growth of Helicobacter pylori: a possible explanation of the effect on peptic ulcer. Gut 35, 1557-1561.

Thompson, L., Edwards, R., Greenwood, D. \& Spiller, R. C. (1992). Possible mechanisms of alterations in colonic flora by diets high in polyunsaturated fatty acids. In Medical and Environmental Aspects of Anaerobes, pp. 144-148 [B. I. Duerden, J. S. Brazier, S. V. Seddon and W. G. Wade, editors]. Petersfield: Wrightson Biomedical Publishing Ltd. 